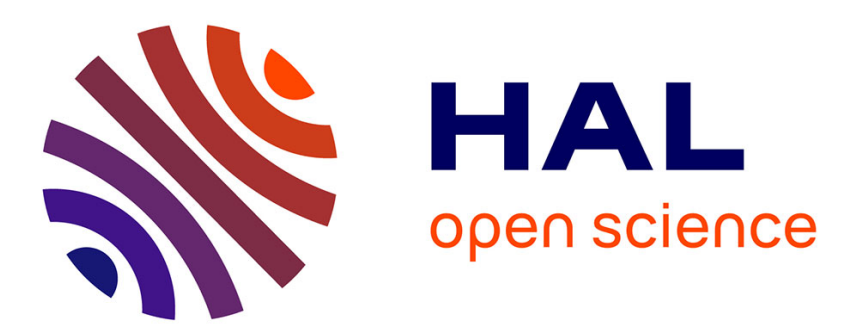

\title{
Phénomènes de piézoconductivité dans les composites polymère-noir de carbone. Application aux capteurs
}

\author{
J.P. Reboul, Ph. Malacan, A. Toureille, S. Hadjri-Mebarki, P. Merle
}

\section{To cite this version:}

J.P. Reboul, Ph. Malacan, A. Toureille, S. Hadjri-Mebarki, P. Merle. Phénomènes de piézoconductivité dans les composites polymère-noir de carbone. Application aux capteurs. Revue de Physique Appliquée, 1990, 25 (4), pp.347-352. 10.1051/rphysap:01990002504034700 . jpa-00246193

\section{HAL Id: jpa-00246193 https://hal.science/jpa-00246193}

Submitted on 1 Jan 1990

HAL is a multi-disciplinary open access archive for the deposit and dissemination of scientific research documents, whether they are published or not. The documents may come from teaching and research institutions in France or abroad, or from public or private research centers.
L'archive ouverte pluridisciplinaire HAL, est destinée au dépôt et à la diffusion de documents scientifiques de niveau recherche, publiés ou non, émanant des établissements d'enseignement et de recherche français ou étrangers, des laboratoires publics ou privés. 


\title{
Phénomènes de piézoconductivité dans les composites polymère-noir de carbone. Application aux capteurs
}

\author{
J. P. Reboul, Ph. Malacan, A. Toureille, S. Hadjri-Mebarki et P. Merle \\ Laboratoire d'Electrotechnique de Montpellier, Université des Sciences et Techniques du Languedoc, place \\ Eugène Bataillon, 34095 Montpellier Cedex 5, France
}

(Reçu le 6 octobre 1989, révisé le 4 décembre 1989, accepté le 5 janvier 1990)

\begin{abstract}
Résumé. - Certains composites polymère-noir de carbone ont une conductivité qui varie beaucoup avec les contraintes et les déformations. Cette piézoconductivité serait liée à une structure anisotrope, à la déformation élastique des contacts entre noirs de carbone et à l'augmentation du nombre des contacts. Un exemple d'utilisation de ces matériaux pour la réalisation de capteurs destinés au contrôle et à la régulation du trafic routier est envisagé.
\end{abstract}

\begin{abstract}
The electrical conductivity of some carbon black-polymer composites is very much affected by an applied mechanical stress or by the resulting strain. Such a piezoconductive behaviour would be concerned with the anisotropic structure of the material, with the mechanics of elastic contact between carbon black particles and with the increase of contact number. As an example, the use of these materials for making sensors useful in road traffic monitoring is discussed.
\end{abstract}

\section{Introduction.}

Les applications des composites polymère-noir de carbone sont de plus en plus nombreuses. La plus ancienne est la réalisation de matériaux antistatiques capables d'écouler les charges et évitant l'accumulation d'électricité statique : les étincelles, les décharges désagréables, les dangers d'explosion qui peuvent en résulter en présence de gaz ou de poussières inflammables sont ainsi évités. Dans les câbles, ces composites sont également utilisés pour canaliser des équipotentielles et éviter l'amorçage de décharges corona qui limitent la durée de vie des isolants. Plus récemment, on a fait appel à ces matériaux pour la protection, contre le rayonnement électromagnétique, des circuits sensibles et des dispositifs de la microélectronique.

Pour que le composite soit conducteur (on dit plus modestement «semiconducteur»), le taux de noir de carbone incorporé à la matrice isolante que constitue le polymère doit être élevé, en général audessus de $30 \%$ en volume, de façon qu'un réseau de mailles conductrices puisse s'établir à travers toute la structure du matériau.

Les polymères chargés ainsi obtenus ont très souvent un comportement non ohmique qui a été expliqué par de la charge d'espace au voisinage des contacts $[1,2]$ et par de l'effet tunnel en volume [3]. Mais quelques-uns de ces matériaux ont, en outre, une conductivité apparente qui varie beaucoup, et parfois même de façon paradoxale, avec les contraintes et les déformations auxquelles ils sont soumis.

Dans le présent travail, nous donnons une explication des mécanismes responsables de ce phénomène de « piézoconductivité ». Par ailleurs, nous montrons les possibilités d'utilisation de ces matériaux pour la réalisation de capteurs et autres dispositifs de détection, en particulier dans les domaines qui concernent le comptage des véhicules et la régulation du trafic routier.

Les polymères composites à propriétés piézoconductrices.

Les polymères chargés de noir de carbone ne présentent pas, en général, de phénomènes de piézoconductivité. Ces propriétés apparaissent seulement sur quelques types de composites et à des degrés assez faibles. Par contre, nous avons trouvé un phénomène très amplifié sur des rubans semiconducteurs utilisés en câblerie, lors des opérations de raccordements de câbles haute-tension. Les proprié- 
tés recherchées dans ces rubans concernent la conductivité, qui doit être suffisante, et le pouvoir autoamalgamant qui permet aux diverses couches de se souder entre elles et de constituer une coquille compacte, une fois enroulées sur le câble. En aucun cas les propriétés piézoconductrices ne sont nécessaires ; elles ne sont pas recherchées et elles apparaissent dans ce type de matériau de façon purement fortuite, en relation avec la composition et les procédés de fabrication, comme nous allons le voir. Plusieurs fabricants commercialisent des rubans semiconducteurs autoamalgamants. Nous avons vérifié que presque tous présentent des phénomènes importants de piézoconductivité. En ce qui concerne notre étude, elle a porté plus particulièrement sur un produit du commerce appelé «Buterton $\mathrm{Ba} 40$ » $\left(^{1}\right)$.

Les rubans ont une largeur de $18 \mathrm{~mm}$ et une épaisseur de $0,8 \mathrm{~mm}$ environ, mais, au départ, le matériau se présente sous forme de larges feuilles qui sont ensuite découpées en bandes avant d'être commercialisées.

Le matériau, est constitué, pour l'essentiel, d'un polymère isolant (polyisobutylène + matrice EPDM) et d'une charge conductrice (noir de carbone Vulcan XC72). D'après le fabricant, le taux de noir est d'environ $40 \%$.

\section{Propriétés électriques des rubans piézoconducteurs.}

Une étude de la conductivité de ces rubans, sous diverses conditions, nous a révélé un certain nombre de particularités.

Tout d'abord, en plus des légères non-linéarités des caractéristiques courant-tension, phénomène fréquent chez les composites polymère-noir de carbone, nous avons noté une très forte anisotropie :

La conductivité est beaucoup plus grande suivant une direction contenue dans le plan de la feuille que dans la direction perpendiculaire à ce plan. Le rapport des conductivités (mesurées sous quelques volts continus) est de l'ordre de 300 .

Il est raisonnable de penser que lors de l'opération d'incorporation des poudres de noir dans la matrice isolante viscoplastique, les grains ne se répartissent pas de façon uniforme et ont tendance à se concentrer dans les sillons creusés par les peignes du malaxeur, formant ainsi des chaînes ou des inhomogénéités filiformes conductrices, équivalentes à des fibres. Lors des opérations de calendrage et d'étirage qui suivent, ces fibres vont se retrouver orientées de façon préférentielle dans le plan de la feuille et on pourra même avoir une certaine stratification. Dès lors, on conçoit très bien que les courants puissent circuler plus facilement dans une direction parallèle au plan de la feuille que dans une direction perpendiculaire à ce plan.

(1) Fabriqué par Ets Barnier, rue Edouard Branly, 26000 Valence.
Cette anisotropie de conductivité serait à l'origine des variations paradoxales de la résistance longitudinale d'un ruban, quand celui-ci est soumis à un effort de traction, et pourrait également expliquer les phénomènes de piézoconductivité dans la direction transversale.

Dans un travail précédent [4], nous avons déjà décrit les variations très inattendues de la résistance longitudinale d'un ruban lorsque celui-ci est étiré et que par conséquent on a simultanément augmentation de sa longueur $L$ et diminution de sa section $S$.

La relation bien connue $R=\rho L / S$ laisserait prévoir une légère augmentation de la résistance, si la résistivité $\rho$ restait constante. En effet, en faisant intervenir la loi de Hooke sur les déformations élastiques, cette augmentation serait :

$$
\Delta R / R=(\Delta L / L)(1+2 \nu),
$$

$\nu$ étant le coefficient de Poisson qui est voisin de 0,3 pour la plupart des matériaux.

Or, en réalité, on observe une forte diminution de la résistance, soit :

$$
\Delta R / R \simeq-20 \Delta L / L
$$

Nous avons proposé une interprétation qui fait intervenir une augmentation du nombre des contacts entre fibres ou inhomogénéités conductrices en relation avec leur déformation relative, conformément à une loi de percolation mentionnée par Carmona et al. [5] dans le cas de mélanges contenant des fibres conductrices.

Sur le plan pratique, une telle propriété est intéressante et peut conduire à de nombreuses applications, notamment pour la réalisation de jauges d'extensométrie et de capteurs de déformation.

Dans le sens de l'épaisseur, la conductivité de ces rubans semble varier de façon moins paradoxale puisqu'elle augmente avec la pression. Or, comme on sait que ces matériaux sont facilement déformables, on peut penser que la simple déformation élastique est responsable de cette diminution de résistance. Il faut toutefois vérifier les ordres de grandeurs.

On a vu que, d'après la loi de Hooke, on doit avoir :

$$
\Delta R / R \simeq 1,6 \Delta L / L, \text { en prenant } \nu=0,3 .
$$

Expérimentalement, nous avons mesuré les variations relatives $\Delta R / R$ de la résistance transversale d'un disque de composite $\mathrm{Ba} 40$, en fonction de la pression appliquée $p$. Les résultats sont représentés sur la figure 1.

A partir de ces résultats, on peut déduire la variation de $\Delta R / R$ en fonction de $\Delta L / L$. En effet, pour une déformation élastique, on a :

$$
\Delta L / L=-p / E,
$$

$E$ étant le module d'Young. 


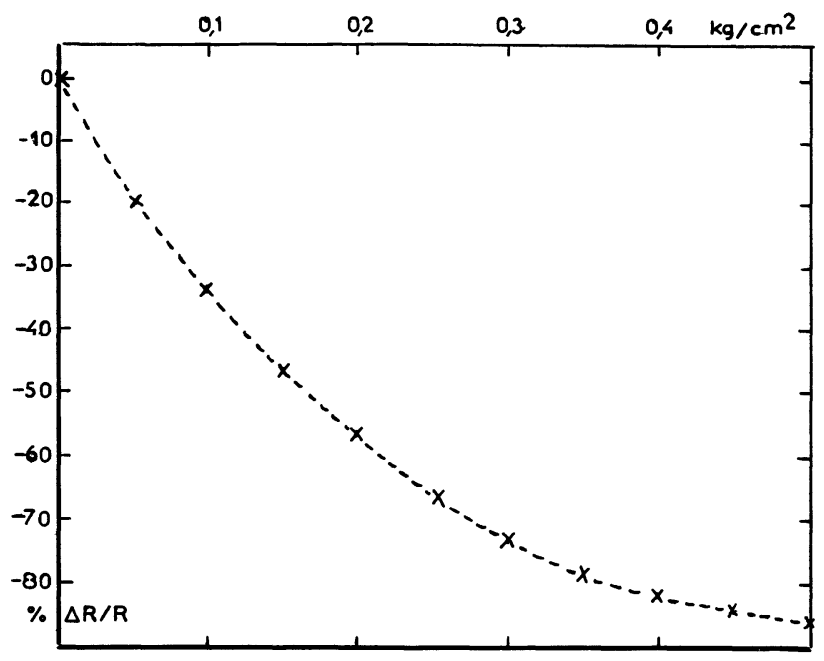

Fig. 1. - Variations relatives de la résistance d'un disque en composite $\mathrm{Ba} 40$, en fonction de la pression appliquée. Dimensions du disque : diamètre $18 \mathrm{~mm}$; épaisseur $0,8 \mathrm{~mm}$.

[Relative resistance variations of a $\mathrm{Ba} 40$ composite disc as a function of the applied strength. Sample dimensions are $18 \mathrm{~mm}$ in diameter and $0.8 \mathrm{~mm}$ in thickness.]

D'après nos mesures, $E \simeq 30 \mathrm{~N} / \mathrm{mm}^{2}$, ce qui donne :

$$
\Delta R / R \simeq 1100 \Delta L / L .
$$

Les variations mesurées expérimentalement sont 600 ou 700 fois plus grandes que celles que donnerait la simple déformation élastique du disque: Il y a donc une augmentation de la conductivité du matériau liée à d'autres phénomènes, comme nous allons tenter de l'expliquer.

\section{Mécanismes possibles intervenant dans la piézocon- ductivité.}

Dans un polymère chargé de noir de carbone, on conçoit très bien qu'une compression puisse augmenter le nombre de contacts entre particules conductrices et par suite rendre le matériau plus conducteur. Ce phénomène est extrêmement important lorsque la concentration en particules conductrices se trouve au voisinage du seuil de percolation. On peut, en effet, assister alors à un véritable basculement entre l'état isolant et l'état conducteur en faisant varier très légèrement le nombre des contacts, par exemple en modifiant la température ou la pression.

Lorsque la concentration en particules conductrices est nettement au-dessus du seuil de percolation et que le composite est déjà franchement conducteur, ce qui est notre cas, l'augmentation du nombre des contacts fait augmenter la conductivité de façon beaucoup plus relative. Pour expliquer une aussi forte variation de la conductivité avec la pression, il faut peut-être faire appel à un autre mécanisme dont l'effet se combinerait à l'augmentation du nombre des contacts : par exemple, l'écrasement élastique des particules conductrices l'une contre l'autre, sous l'effet de la compression, et par suite, l'augmentation des surfaces en contact et la diminution des résistances. La matrice isolante étant très déformable, ce phénomène doit exister nécessairement.

Plusieurs auteurs ont étudié la variation de la surface de contact entre deux solides en présence d'un revêtement constitué par un autre matériau et lorsqu'ils sont soumis à une force de compression. Leveson [6] étudie l'aspect mécanique du contact entre deux solides de forme sphérique ou cylindrique, en présence d'un lubrifiant. Vandamme [7] étudie la variation de la résistance et du bruit de fond, en fonction de la force appliquée, dans un contact entre deux semiconducteurs recouverts d'un film d'oxyde.

De tels modèles nous ont paru applicables à des particules de noir de carbone enrobées de polymère isolant. Toutefois, on ne peut envisager que des contacts directs entre particules, car la présence d'une couche de polymère isolant entre deux grains constitue un obstacle au passage des électrons, sauf, peut-être, par effet tunnel à tension élevée, ce qui n'est pas notre cas.

Vandamme [7] fait intervenir la surface de contact entre deux particules sphériques qui a été calculée par Hertz et dont le rayon vaudrait, en prenant la valeur 0,3 pour le coefficient de Poisson :

$$
a=0,88(F r / E)^{1 / 3},
$$

$F$ est la force de compression entre les deux particules, $r$ leur rayon et $E$ le module d'Young.

Par ailleurs, comme la résistance du contact est donnée par la relation $R=\rho / \pi a, \rho$ étant la résistivité des particules et que, d'autre part, $F=p \pi r^{2}$, on aboutit à :

$$
R=\rho E^{1 / 3} / 0,88 \pi^{4 / 3} p^{1 / 3} r .
$$

La conductance du contact est donc de la forme :

$$
g=A p^{1 / 3} r
$$

$A$ étant une constante.

$\mathrm{Si}$ on admet une structure stratifiée du matériau, ce que semble indiquer l'anisotropie de conductivité, la conduction dans la direction perpendiculaire aux strates va faire intervenir $\boldsymbol{n}$ contacts en parallèle entre deux strates successives. D'où une conductance :

$$
g_{n}=A p^{1 / 3}\left(r_{1}+r_{2}+\cdots+r_{n}\right),
$$

soit, en faisant intervenir le rayon moyen des particules :

$$
g_{n}=A p^{1 / 3} n i
$$


Cette formule donne une conductance nulle pour $p=0$, ce qui est contraire à la réalité. On doit donc admettre l'existence d'un certain nombre de chaînes ou de contacts «soudés» ayant une conductance $g_{0}$ en l'absence de pression et indépendante de celleci de sorte que :

$$
g_{n}=g_{0}+A p^{1 / 3} n \imath .
$$

S'il y a $n^{\prime}$ interfaces identiques entre une électrode et l'autre, la conductance de l'éprouvette sera :

$$
G=g_{0} / n^{\prime}+A i n p^{1 / 3} / n^{\prime},
$$

expression de la forme :

$$
G-G_{0}=\alpha n p^{1 / 3} .
$$

Dans une première approche, on peut supposer $n$ indépendant de $p$. On obtient alors :

$$
\log \left(G-G_{0}\right)=K+\log p / 3 .
$$

Sur la figure 2, nous avons représenté $\log \left(G-G_{0}\right)$ en fonction de $\log p$, pour un disque de $\mathrm{Ba} 40$. On peut noter que la pente de la courbe est partout supérieure à $1 / 3$ et que c'est seulement vers les faibles pressions qu'elle tend vers cette valeur.

Il faut donc admettre que $n$ n'est pas une constante mais une fonction croissante de la pression $p$, ce qui se traduit par l'écart entre la courbe expérimentale et la droite de pente 1/3. Mais l'étude de cet écart doit permettre de cerner d'un peu plus près la fonction $n_{(p)}$ représentant le nombre de contacts en fonction de la pression.

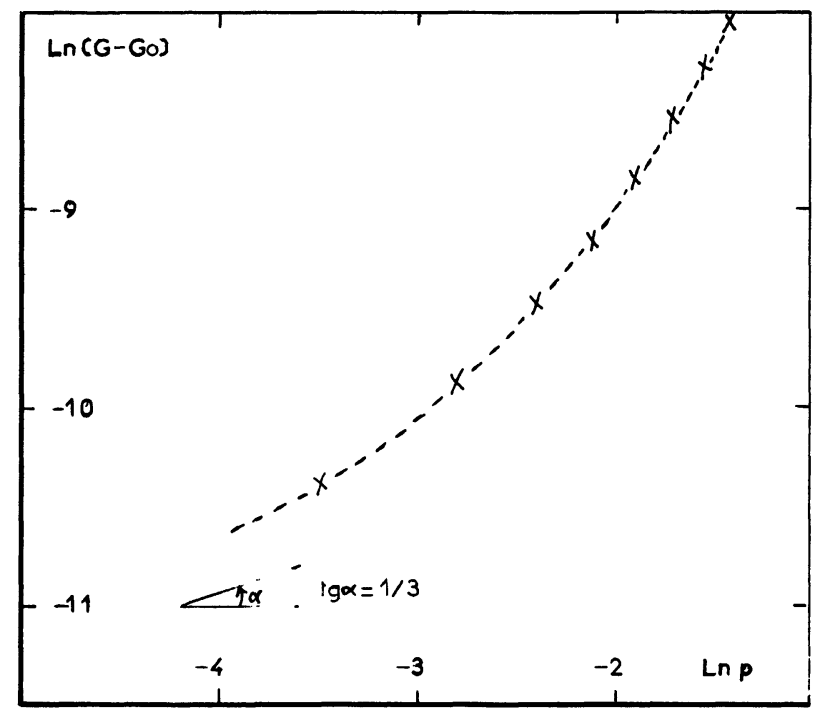

Fig. 2. - Augmentation de la conductance en fonction de la pression appliquée (éprouvette de la Fig. 1).

[Increase of the sample conductance as a function of the applied strength (sample of Fig. 1).]
Sur la figure 3, nous avons tracé, en fonction de $p$, la quantité $\left(G-G_{0}\right) p^{-1 / 3}$, c'est-à-dire $\alpha n_{(p)}$, d'après la relation (1).

En extrapolant la courbe vers $p=0$, on peut déduire l'ordonnée à l'origine :

$$
\alpha n_{(0)}=0,8 \times 10^{-4} .
$$

On peut alors, pour chaque point de la courbe, calculer le rapport $\alpha n_{(p)} / \alpha n_{(0)}=n / n_{0}$ et ensuite, représenter $\log \left(n / n_{0}\right)$ en fonction de la pression $p$. Les résultats sont représentés sur la figure 4 . On obtient des point alignés sur une droite passant par l'origine, de pente $\beta=8,2$ pour des pressions exprimées en $\mathrm{kg} / \mathrm{cm}^{2}$.

Le nombre de contacts obéirait donc, du moins pour les faibles pressions, à une loi du type $n=n_{0} \exp (\beta p)$, ce qui signifie physiquement que l'augmentation du nombre des contacts est proportionnelle au nombre des contacts existants $(d n=K n \mathrm{~d} p)$.

La structure du noir de carbone pourrait expliquer une loi de ce type.

Le noir Vulcan XC-72 (ou N 472) utilisé ici est reconnu hautement conducteur et employé comme tel. Ses particules plus ou moins sphériques, de l'ordre de la dizaine ou de quelques dizaines de $\mathrm{nm}$, sont associées en agrégats étalés et structurés atteignant le $\mu \mathrm{m}$ [8]. Il est donc raisonnable de penser que, pour un même agrégat, plusieurs contacts supplémentaires puissent prendre naissance autour d'un premier contact, au cours de la compression.

En conséquence, il semble que la prise en compte simultanée de la diminution des résistances de contact et de l'augmentation du nombre des contacts, phénomènes qui doivent obligatoirement coexister dans ces matériaux, fournisse une approche convenable des mécanismes de piézoconductivité.

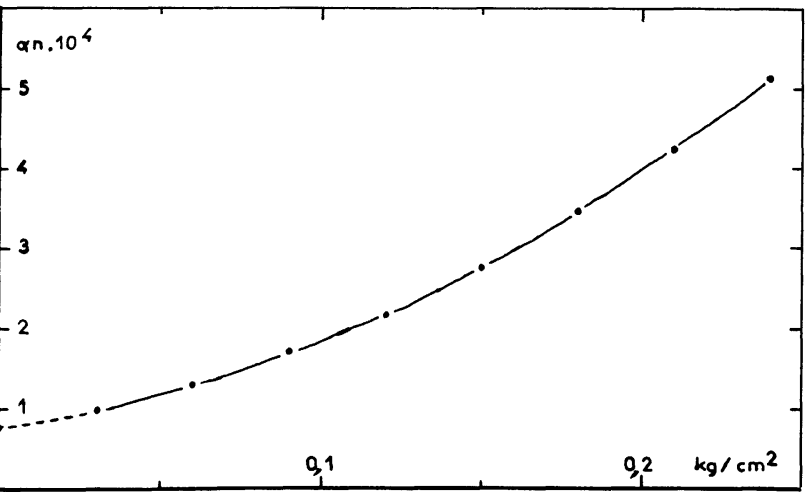

Fig. 3. - Augmentation du nombre de contacts entre strates en fonction de la pression appliquée (éprouvette de la Fig. 1).

[Increase of the number of contacts between layers as a function of the applied strength (sample of Fig. 1).] 


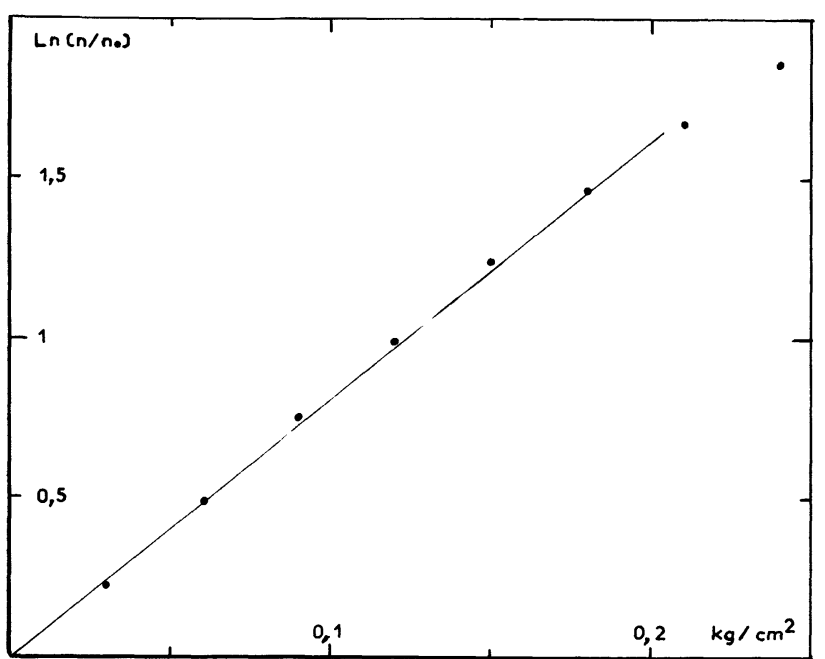

Fig. 4. - Variation de $n / n_{0}$ en fonction de la pression appliquée (éprouvette de la Fig. 1).

[Variation of $n / n_{0}$ as a function of the applied strength (sample of Fig. 1).]

\section{Applications.}

Les domaines possibles d'applications des composites piézoconducteurs sont nombreux : jauges d'extensométrie, capteurs de contrainte et de déplacement, détecteurs de passage, comptage, reconnaissance de formes, touches et interrupteurs sensibles, peau artificielle pour la robotique [9].

Nous nous sommes intéressé plus particulièrement à leur possibilité d'utilisation pour la réalisation de détecteurs de passage de véhicules dans les dispositifs de comptage et de régulation du trafic routier. Les détecteurs actuels sont loin de donner satisfaction. Les uns sont fragiles et peu fiables, comme par exemple les tubes pneumatiques. D'autres nécessitent d'importants travaux de génie civil pour leur mise en place, comme par exemple les boucles électromagnétiques. Le plus récent, le câble coaxial piézoélectrique [10] est cher; son étalonnage est délicat; il nécessite un matériel sophistiqué et des équipes spécialisées. La réalisation d'un capteur routier peu coûteux et facile à mettre en place, par exemple par collage sur la chaussée, présente donc un intérêt économique certain. Les polymères chargés de noir de carbone pourraient trouver là une nouvelle application en permettant la réalisation de dispositifs dont l'impédance chute chaque fois qu'ils sont franchis par un véhicule. En effet, en alimentant un tel dispositif sous quelques volts, en série avec une résistance, on obtient des impulsions de tension aux bornes de la résistance.

Les premiers essais que nous avons réalisés sont encourageants. La figure 5 représente les impulsions de tension que nous avons obtenues, lors du passage d'une voiture et d'une bicyclette, avec un capteur utilisant le composite $\mathrm{Ba} 40$.

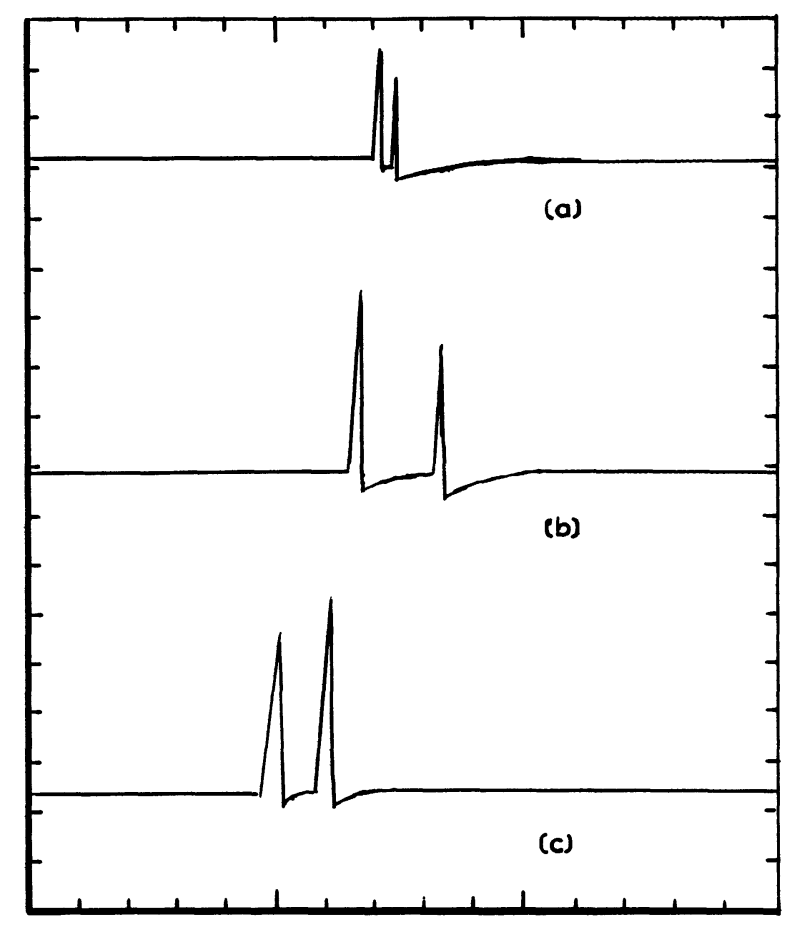

Fig. 5. - Enregistrements des impulsions de tension données par un détecteur de passage utilisant le composite $\mathrm{Ba} 40$. Horizontalement, 1 div. $=0,4$ seconde. Verticalement, 1 div. = 100 millivolts. (a) Voiture passant à $90 \mathrm{~km} / \mathrm{h}$. (b) Voiture passant à $15 \mathrm{~km} / \mathrm{h}$. (c) Bicyclette passant à $10 \mathrm{~km} / \mathrm{h}$.

[Graphs of voltage pulses obtained with a $\mathrm{Ba} 40$ composite sensor when crossed by vehicles : horizontally : $0.4 \mathrm{~s} / \mathrm{div}$, vertically : $100 \mathrm{mV} /$ div. (a) Car driven at $90 \mathrm{~km} / \mathrm{h}$ speed. (b) Car driven at $15 \mathrm{~km} / \mathrm{h}$ speed. (c) Bike at $10 \mathrm{~km} / \mathrm{h}$.]

Mais pour être exploitables, ces dispositifs doivent répondre à un cahier des charges en ce qui concerne la sensibilité et le temps de réponse. En effet, ils doivent être capables de détecter le passage d'un poids lourd, d'une voiture ou d'une bicyclette, avec des plages de vitesse aussi différentes. De plus, il serait souhaitable qu'ils puissent permettre le classement catégoriel des véhicules.

Une étude de faisabilité d'un capteur routier à partir d'un polymère chargé est actuellement en cours au Laboratoire [11]. Elle porte sur la mise au point d'un matériau piézoconducteur stable et fiable, sur la conception de sa structure, sur la détermination de la géométrie du capteur et des électrodes, de façon à avoir les sensibilités et les temps de réponse compatibles avec ce genre d'utilisation.

\section{Conclusion.}

Dans ce travail, nous avons donné une approche des mécanismes responsables du comportement piézoconducteur de certains composites polymère-noir de carbone. Une structure très anisotrope et stratifiée semble être à l'origine du phénomène. De façon plus 
précise, l'augmentation de conductivité serait liée, d'une part, à la déformation élastique des contacts entre particules, donnant des variations de conductance en $p^{1 / 3}$, d'autre part, à l'augmentation du nombre des contacts suivant une loi exponentielle, en relation avec la structure du noir de carbone.
Une fois les mécanismes de la piézoconductivité des composites mieux connus et mieux compris, une nouvelle classe de matériaux plus performants, aux propriétés stables et reproductibles devrait voir le jour, favorisant ainsi toute une gamme de nouvelles applications.

\section{Bibliographie}

[1] Reboul J. P., J. Appl. Phys. 46 (1975) 2961.

[2] Reboul J. P., Moussalli G., Intern. J. Polym. Mat. 5 (1976) 133.

[3] Sichel E. K., Gittleman J. I., Carbon BlackPolymer Composites, Ed. E. K. Sichel (Dekker, New York) 1982.

[4] Reboul J. P., Abou-DakKa M., Toureille A., Rev. Gen. Elect. 4 (1988) 45 ; 6 (1988) 54.

[5] Carmona F., Delaes P., Barreau F., Ordiera D., Canet R., Lafeychine L., Rev. Chim. Min. 18 (1981) 498.
[6] Leveson R. C., J. Appl. Phys. 45 (1974) 1041.

[7] Vandamme L. K. J., J. Appl. Phys. 45 (1974) 4563.

[8] Medalia A. I., Carbon Black, Polymer Composites, Ed. E. K. Sichel (Dekker, New York) 1982.

[9] Clot J., Falipou J., Micro-systèmes (septembreoctobre 1982) p. 67.

[10] MANSION D., Le Nouvel Automatisme (mars 1982) p. 68.

[11] Malacan Ph., Mémoire CNAM (en préparation). 